\title{
Intractable Hiccups Due to Posterior Inferior Cerebellar Artery Aneurysm
}

\author{
Monzer Chehab ${ }^{1}$ Samir Noujaim ${ }^{1} \quad$ Omar Qahwash ${ }^{2}$ Duane Mezwa ${ }^{1}$ Anindya Roy ${ }^{1}$ \\ ${ }^{1}$ Department of Radiology, Oakland University William Beaumont \\ School of Medicine, Royal Oak, Michigan, United States \\ 2 Department of Neurosurgery, Oakland University William Beaumont \\ School of Medicine, Royal Oak, Michigan, United States \\ Address for correspondence Monzer Chehab, MD, Department of \\ Radiology, Oakland University William Beaumont School of Medicine, \\ 3601 W. 13 Mile Rd. 2 Center, Royal Oak, MI 48073, United States \\ (e-mail: moe.chehab@beaumont.edu).
}

J Neurol Surg Rep 2015;76:e120-e122.

Abstract
Keywords
- intractable hiccups
- PICA aneurysm
- MRA

This is the third reported case of a posterior inferior cerebellar artery (PICA) aneurysm presenting as intractable hiccups $(\mathrm{IH})$. A previously healthy 29 -year-old woman was admitted with a 2-week history of hiccups occurring $>100$ times per minute. Symptoms persisted despite numerous noninvasive therapies. Magnetic resonance imaging and magnetic resonance angiogram of the brain showed a left PICA aneurysm that was confirmed by catheter angiography. Symptoms resolved following suboccipital craniotomy and resection. Although rare, PICA aneurysm is a potentially curable cause of $\mathrm{IH}$.

\section{Introduction}

Hiccups, or singultus, are the result of the involuntary myoclonic contracture of the diaphragm. ${ }^{1}$ In the setting of a closed airway, the contracture causes the peculiar sound typical of the condition. ${ }^{1}$ Hiccups are characterized as intractable when they occur 40 to 100 times per minute for $>48$ hours. $^{2}$ The underlying cause of intractable hiccups $(\mathrm{IH})$ is broad, ranging from idiopathic to gastrointestinal to central nervous system lesions involving the brainstem. ${ }^{3}$ We present a case of IH successfully treated by surgical resection of a posterior inferior cerebellar artery (PICA) aneurysm in a previously healthy 29 -yearold woman.

\section{Case Report}

A 29-year-old woman was admitted with a 2-week history of hiccups occurring up to 100 times per minute. She was seen by her primary care physician 1 week prior who prescribed Thorazine and metoclopramide with no significant improvement. She had no prior medical or surgical history and denied any history of anxiety, depression, or psychosis. Vital signs were within normal limits, and physical examination was nonfocal. A detailed neurologic examination revealed no focal weakness, loss of sensation, or imbalance. Chest X-ray, contrast-enhanced computed tomography scan of the chest, abdomen, and pelvis, and upper endoscopy were negative. For 5 days, symptoms had a waxing and waning course on chlorpromazine, metoclopramide, and baclofen. Magnetic resonance imaging (MRI) of the brain demonstrated a partially enhancing $1.3 \times 1.1-\mathrm{cm}$ mass within the fourth ventricle causing mass effect on the medulla oblongata ( - Fig. 1A). Magnetic resonance angiogram (MRA) of the cerebral circulation demonstrated that the mass was in fact a partially thrombosed aneurysm ( - Fig. 1B). Catheter angiogram confirmed the presence of dolichoectatic aneurysm of the distal left posterior inferior cerebellar artery (-Fig. 2). The following day, the patient underwent a midline suboccipital craniotomy with resection of aneurysm and primary anastomosis. Pathologic evaluation of the resected specimen demonstrated a partially thrombosed aneurysm (-Fig. 3). Her symptoms resolved immediately, and her hospital course was uncomplicated. She was discharged on postoperative day 6 in stable condition.

\section{Discussion}

This is the third reported case of IH caused by a giant PICA aneurysm. The first reported case was in 2007, of 16-year-
License terms

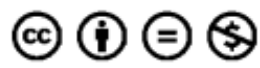

received

August 12, 2014

accepted

February 1, 2015

published online

April 27, 2015
DOI http://dx.doi.org/

$10.1055 / \mathrm{s}-0035-1549221$. ISSN 2193-6366. (c) 2015 Georg Thieme Verlag KG
Stuttgart · New York 


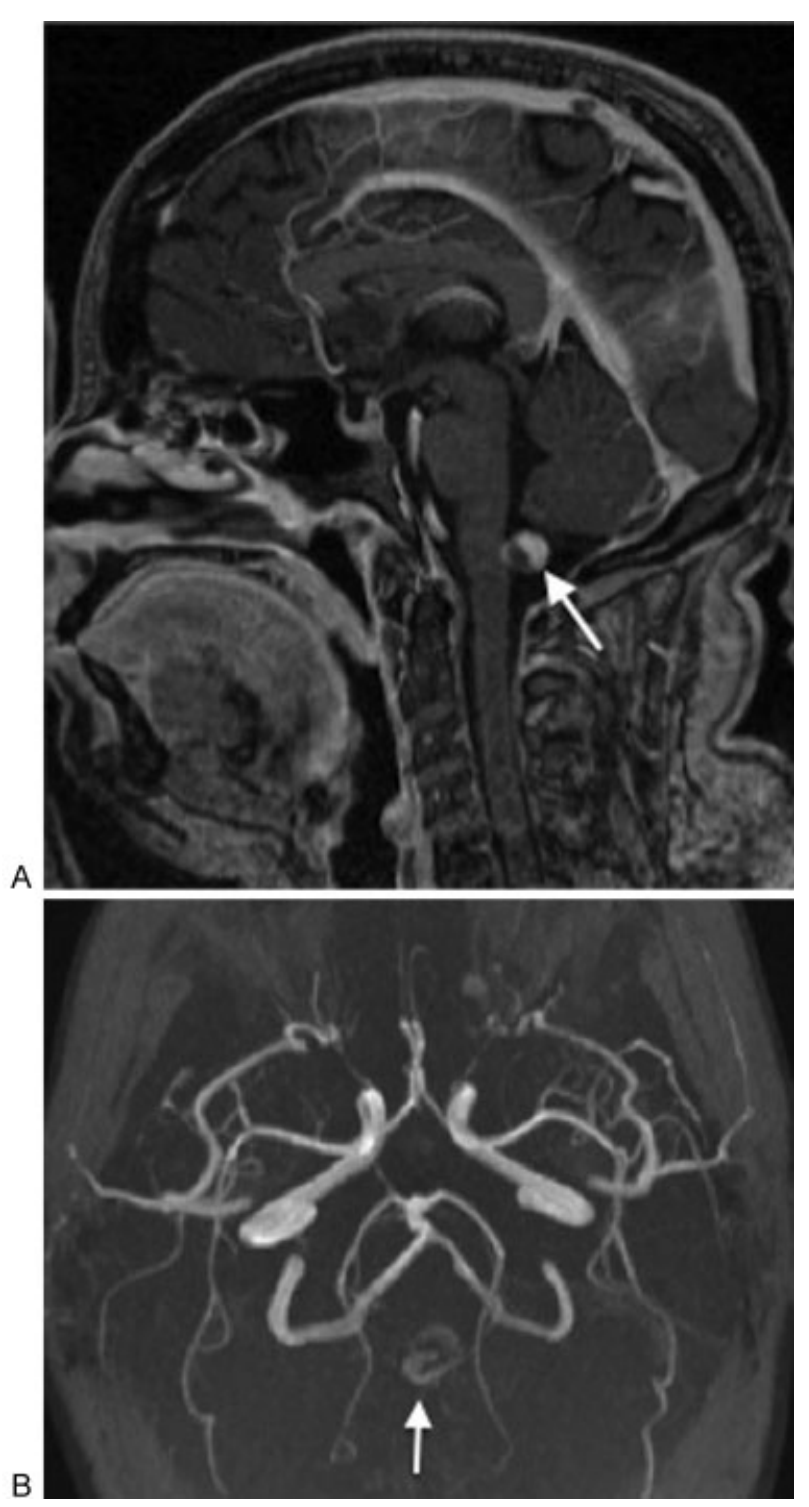

Fig. 1 (A) Sagittal contrast-enhanced magnetic resonance imaging and (B) axial magnetic resonance angiogram of the brain demonstrate a partially thrombosed posterior inferior cerebellar artery aneurysm within the fourth ventricle (arrows).

old boy with 1-day history of $\mathrm{IH}^{4}{ }^{4}$ The other case was reported in 2010 of a 23-year-old woman who had a 3-month history of occasional hiccups that suddenly became intractable just before admission. ${ }^{5}$ All three patients were relatively young, healthy, with nonfocal neurologic exams, yet debilitating IH symptoms refractory to noninvasive therapy. All three were diagnosed following contrast-enhanced MRI of the brain that showed a partially thrombosed, unruptured aneurysm of the distal PICA occupying the fourth ventricle at the level of the medulla. Our patient was the only one who underwent preoperative cerebral angiogram, used to confirm a vascular origin and provide a roadmap before surgical resection. All three patients had complete symptom resolution following surgical resection and primary anastomosis via a suboccipital approach.

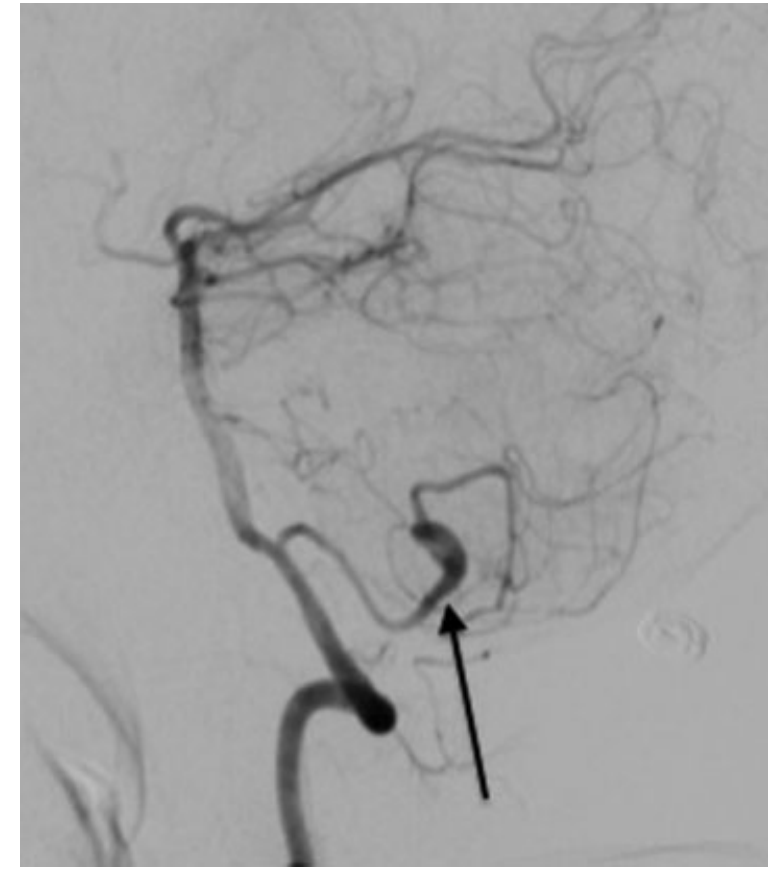

Fig. 2 Digital subtraction angiogram in the sagittal projection obtained following contrast injection into the left vertebral artery confirmed a partially thrombosed dolichoectatic aneurysm of the left distal posterior inferior cerebellar artery (arrow).

There are numerous causes of IH. Most are due to gastric distention and/or gastroesophageal reflux. ${ }^{2}$ IH occurring with systemic illnesses such as sepsis or uremia have been described, in addition to psychiatric disorders such as hysteria, anorexia, or grief. ${ }^{4}$ Infrequently, a peripheral nervous system cause of $\mathrm{IH}$ is possible as the result of irritation along the hiccup reflex arch including the thoracic sympathetic chain, phrenic nerve, or accessory respiratory musculature. ${ }^{1}$ Even more rare are lesions of the central nervous system that occur as the result of an insult to the "hiccup center" in the medulla oblongata. ${ }^{6}$ Ischemia

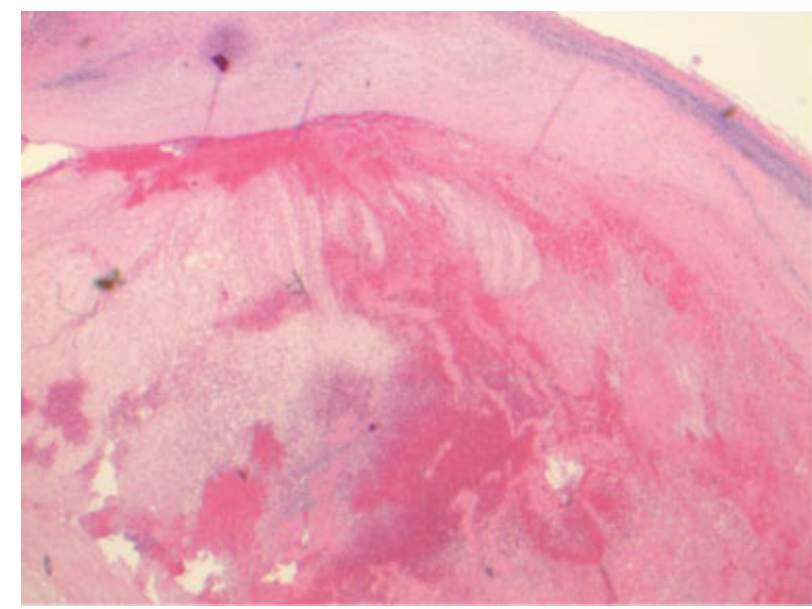

Fig. 3 Hematoxylin and eosin stained micrograph at $\times 200$ magnification shows an organized thrombus within the resected vessel (arrow). 
and mass effect from tumors such as cavernoma, ependymoma, or choroid plexus tumor have been described, occurring in higher incidences than aneurysms. ${ }^{5,6}$ It is therefore prudent to exclude central nervous system lesions in patients presenting with $\mathrm{IH}$ utilizing contrastenhanced MRI with MRA. ${ }^{7}$ Given the propensity of aneurysms to thrombose, differentiation from a hemorrhagic mass may be difficult, and confirmation with cerebral angiography should be considered.

PICA aneurysms make up only $0.6 \%$ of all intercranial aneurysms. ${ }^{8}$ Although IH is an extremely uncommon presentation of a PICA aneurysm, it offers a potentially curable etiology and could eliminate the risk for complications of PICA rupture such as subarachnoid hemorrhage or infarction of the lateral medulla (i.e., Wallenberg syndrome). ${ }^{9}$

Treatment of IH should begin with vagal maneuvers such as breath holding, immersion in cold water, or application of supraorbital pressure. ${ }^{6}$ Persistent hiccups indicate the use of pharmacologic agents such as chlorpromazine, metoclopramide, gabapentin, orphenadrine, or antidepressants. ${ }^{10}$ Hiccups resistant to noninvasive treatment necessitates a search for an underling cause in which case the possibility of brainstem lesions, including aneurysms, should not be overlooked.

\section{References}

1 Fodstad H, Nilsson S. Intractable singultus: a diagnostic and therapeutic challenge. Br J Neurosurg 1993;7(3):255-260

2 Federspil PA, Zenk J. Singultus. HNO 1999;47(10):867-875

3 Chang F-Y, Lu C-L. Hiccup: mystery, nature and treatment. J Neurogastroenterol Motil 2012;18(2):123-130

4 Amirjamshidi A, Abbassioun K, Parsa K. Hiccup and neurosurgeons: a report of 4 rare dorsal medullary compressive pathologies and review of the literature. Surg Neurol 2007;67(4):395-402; discussion 402

5 Gambhir S, Singh A, Maindiratta B, Jaeger M, Darwish B, Sheridan M. Giant PICA aneurysm presenting as intractable hiccups. J Clin Neurosci 2010;17(7):945-946

6 Ferdinand P, Oke A. Intractable hiccups post stroke: case report and review of the literature. J Neurol Neurophysiol 2012;3(5):140

7 Peluso JP, van Rooij WJ, Sluzewski M, Beute GN, Majoie CB. Posterior inferior cerebellar artery aneurysms: incidence, clinical presentation, and outcome of endovascular treatment. AJNR Am J Neuroradiol 2008;29(1):86-90

8 Lehto $H$, Harati A, Niemelä $M$, et al. Distal posterior inferior cerebellar artery aneurysms: clinical features and outcome of 80 patients. World Neurosurg 2014;82(5):702-713

9 Moretto EN, Wee B, Wiffen PJ, Murchison AG. Interventions for treating persistent and intractable hiccups in adults. Cochrane Database Syst Rev 2013;1:CD008768

10 Marsot-Dupuch K, Bousson V, Cabane J, Tubiana JM. Intractable hiccups: the role of cerebral MR in cases without systemic cause. AJNR Am J Neuroradiol 1995;16(10):2093-2100 